Article

\title{
Real-Time Dispatch of Coupled Transmission and Distribution Systems within a Distributed and Cooperative Framework
}

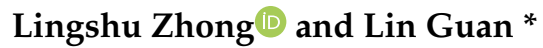 \\ Department of Electric Power, South China University of Technology, Guangzhou 510641, China; \\ z.lingshu@mail.scut.edu.cn \\ * Correspondence: lguan@scut.edu.cn
}

Received: 18 September 2020; Accepted: 3 October 2020; Published: 12 October 2020

check for updates

\begin{abstract}
This paper presents a real-time dispatch strategy for coupled transmission and distribution systems within a distributed and cooperative control framework to maintain reliable and secure operation with minimum generation costs and maximum renewable energy consumption. The presented strategy transforms each distribution system into a dispatchable active source via an average-consensus-based active power control of renewable distributed energy resources (DERs) at the distribution level and then dispatches the active power reference of conventional generators as well as the distribution systems in a measurement-based way at a transmission level. The voltage fluctuation caused by the DER active power control is smoothened with a distributed voltage control method, which can also reduce the active power loss in the distribution systems. Compared to existing real-time dispatch strategies, the proposed strategy can eliminate security issues in the transmission system in a short time by regulating large amounts of DERs at a distribution level in a simple and easy controlled structure, in which the differences in the communication conditions and privacy requirements between the distribution and transmission systems are adequately considered.
\end{abstract}

Keywords: real-time dispatch; distributed optimization; security-constrained economic dispatch; renewable energy resources

\section{Introduction}

With the growing penetration of renewable distributed energy resources (DERs) in distribution systems, the uncertain active power injection of DERs may be reflected in the net load fluctuations at the transmission level, which can deflect the operating points of the transmission system from the schedules and lead to security problems [1-3]. Real-time dispatch [4,5] has been proposed to adjust these deviations based on measurements to maintain a secure operation, but the low ramp rate of conventional dispatchable generators at the transmission level limits their ability to rapidly respond to uncertain fluctuations and eliminate security risks on short notice.

To solve this problem, a promising approach is to integrate the active power control of DERs into the real-time dispatch framework to fully exploit the high-ramp-rate inverters of DERs [6,7]. In particular, appropriate DER active power curtailment can effectively alleviate branch overloads by avoiding excessive power injection from distribution systems. However, to achieve the above, the following difficulties must be overcome.

\subsection{The Balance between Regulation Speed and Regulation Cost}

Maximizing the consumption of clean and inexpensive electrical power provided by DERs can decrease the use of fossil fuels and the emission of greenhouse gases in power generation [8]. In this 
case, although DERs can enable fast and flexible adjustments, it is too costly from economic and environmental perspectives to use substantial DER active power curtailment for long periods of time to maintain secure operations. As a balance between regulation speed and regulation cost, the active power of DERs should be gradually re-consumed and the system load profile gradually changed by small-ramp-rate conventional generators right after security problems have been quickly resolved by the DER active power curtailment. In this case, coordination between the DERs in the distribution systems and the conventional generators in the transmission system is necessary.

\subsection{The Compromise between Real-Time Performance and the Ability to Handle Complexity}

The real-time dispatch for a coupled transmission and distribution system is a large-scale problem for two reasons. On the one hand, a large amount of low-power DERs scattered in the distribution systems need to be regulated to produce an adequate change in the transmission power flow, which will substantially increase the decision variables of the dispatch problem. On the other hand, the coupled transmission and distribution system model needs to describe every load bus at the transmission level as a detailed distribution system, which will also increase the model complexity. A distributed optimization technique is used to handle these complexities and make the coordination dispatch problem solvable on a relatively longer timescale $[9,10]$, but a lighter approach that does not require the solution of large-scale optimization problems is still needed to deal with the dispatch problem on a real-time scale.

\subsection{The Differences in the Communication Conditions and Privacy Risks between the Transmission and Distribution Systems}

There is a large gap in the degree of informatization between the distribution and transmission systems in that many advanced measurement and communication infrastructures that are common in transmission systems are too expensive to be used in distribution systems. For this reason, some promising real-time dispatch strategies may face a measurement or communication infrastructure shortage while considering the details of distribution systems. As an example, measurement-based dispatch strategies such as those in [4] can solve the transmission dispatch problem in real time considering the N-1 line-loading security indicators, but they require all the buses in the system to be measurable by phasor measurement units (PMU) to achieve a complete wide-area measurement. This requirement is possible as technology advances and the cost of measuring equipment decreases in transmission systems, but it will take a much longer time for distribution networks, especially for medium- and low-voltage distribution networks that are widely connected with DERs, to meet this standard.

Moreover, real-time load demand measurement in low-voltage distribution systems is much more sensitive than in transmission systems because individual behavioral information can be analyzed based on personal power consumption data, which may correspond to a single bus in the distribution system [11].

In this case, a reasonable scheme for the real-time dispatch coordination of distribution and transmission systems should fully consider the disparate measurement and communication conditions as well as the privacy risks in these systems to take advantage of the relatively rich information infrastructure on the transmission level while adapting to the relative lack of communication and measurement in distribution networks.

\subsection{The Risk of Voltage Violation in Distribution Systems Caused by Active Power Control}

Due to the high impedance ratio of distribution networks, a rapid change in the DER active power will lead to a large voltage deviation at the distribution level. If there is no appropriate reactive power control strategy to coordinate with the active power dispatch, the distribution networks may suffer their own voltage security problems while adjusting DERs to support the transmission system. 
To overcome these difficulties, a series of studies has sprung up, for example, Ref [12] proposes a process to dispatch the operation of a distribution feeder with heterogeneous prosumers through prosumers data driven forecasting and model predictive control of electrochemical storage, Ref [13] proposes a new cost-effective coordination schemes to drive thousands of non-centrally monitored distributed energy resources to provide voltage support from distribution systems to transmission networks, Ref [14] proposes a hierarchical coordination control strategy for solving the power management problem of an active distribution network with high penetration of distributed generation and make it dispatchable as an adjustable PQ node, and [15] apply a two-dimensional adaptive control scheme known as extremum seeking to enable simultaneous feeder head active power and voltage magnitude reference tracking and feeder voltage regulation without the requirement of the knowledge of the distribution model. These studies successfully combine the compromise between real-time performance and the ability to handle complexity in the distribution systems, but not consider the balance between the regulation speed and regulation cost in the transmission systems.

To obtain the optimal operation for both the transmission and distribution systems, coordinated economic dispatch of transmission and distribution grids is studied, for example, Ref [16] establish and analyze a coordinated transmission and distribution AC optimal power flow model in a master-slave structure, which is solved by a heterogeneous decomposition algorithm inspired by heterogeneous transmission and distribution characteristics to improve economic operations, mitigate voltage rises, and decrease boundary bus mismatch and [17] proposes an affine arithmetic-based interval power flow algorithm of integrated transmission and distribution networks with a novel two-step approximation method for sine and cosine functions, of which effectiveness, conservative property, a fairly good approximation effect, high efficiency, and low data exchange amount are demonstrated by numerical experiments. These optimization-based methods can quickly solve the optimal power flow model problem considering the detailed model of distribution systems, but are not fast enough to guarantee real-time performance.

In this paper, we propose a new coordinated real-time dispatch strategy for coupled transmission and distribution systems, in which a decentralized coordinated control structure is adopted. For each distribution system, an average-consensus-based distributed control structure associated with the limited broadcast communication can rapidly regulate the active power injected into the transmission system to a given reference while sharing the active power of the DERs according to their current generating capacities, maintaining the bus voltages within their limitations by simultaneously regulating the DER reactive power. Moreover, the total DER active power generation and total DER active power curtailment are calculated and submitted to the transmission level for further optimization. In this case, massive DERs in a distribution system can be dispatched as one entity. At the transmission level, a measurement-based centralized real-time dispatch strategy is used to minimize the total generation cost of conventional generators at the transmission level and the total DER active power curtailment at the distribution level while maintaining the N-1 line-loading security. Compared to existing methods, the proposed strategy can: (1) Rapidly solve the security problems at the transmission level with the DERs at the distribution level and then regain the DER active power consumption with conventional generators to maintain an optimal security operation; (2) avoid the direct control of a large number of DERs to enable the dispatch strategy to operate on real-time scale with limited computing resources; (3) make full use of wide-area measurements in the transmission system to avoid the influence of model parameter errors while minimizing the requirements for extra communication infrastructure and private or unmeasurable data in the distribution systems; and (4) alleviate the voltage fluctuation caused by the DER active control with a coordinated reactive power control, which can also reduce the active power loss in the distribution systems.

The remainder of the paper is organized as follows. Section 2 describes the proposed real-time dispatch strategy at the transmission level, while Section 3 describes the strategy at the distribution level. The performance of the proposed method is tested with numerical simulations in Section 4 . Finally, Section 5 concludes the paper by summarizing the findings. 


\section{Real-Time Dispatch Strategy at the Transmission Level}

A typical real-time security-constrained economic dispatch problem is extended on the transmission level in this section. Each distribution system with controllable renewable DERs is modeled as a dispatchable active source without ramp rate limitations, while the total DER active power curtailment is modeled as a penalty term in the objective function. The dispatch models are locally linearized with a measurement-based method using PMU data, which are simple enough to be solved in real-time with a centralized controller.

\subsection{Formulation of Security and Economic Indicators}

Without a loss of generality, the operating cost including the generation cost and total renewable energy curtailment is regarded as the economic indicator to be optimized at the transmission level, while the $\mathrm{N}-1$ line-loading limitations are considered as security constraints. The detailed formulation of the above indicators is as follows:

\subsubsection{Generation Cost}

The total generation cost in the transmission system can be calculated with a cost function of all the conventional generators:

$$
\operatorname{cost}_{G}\left(P_{G}^{\text {tran }}\right)=\sum_{G_{i} \in G^{\text {tran }}} \operatorname{cost}_{G_{i}}\left(P_{G_{i}}^{\text {tran }}\right)
$$

where $P_{G_{i}}^{\text {tran }}$ is the active generation of a conventional generator $G_{i}, G^{\text {tran }}$ is the set of all generators, $P_{G}^{\text {tran }}$ is a column vector of $P_{G_{i}}^{\text {tran }}$ for all the $G_{i} \in G^{\text {tran }}$, and $\operatorname{cost}_{G_{i}}\left(P_{G_{i}}^{\text {tran }}\right)$ is the cost function of $G_{i}$, which can be formulated as:

$$
\operatorname{cost}_{G_{i}}\left(P_{G_{i}}^{\text {tran }}\right)=a_{i}\left(P_{G_{i}}^{\text {tran }}\right)^{2}+b_{i} P_{G_{i}}^{\text {tran }}+c_{i}
$$

where $a_{i}, b_{i}$, and $c_{i}$ are constant coefficients given in advance.

\subsubsection{Renewable Energy Consumption Indicator}

Renewable energy consumption is another important economic indicator. In this study, we assume that only the active power of renewable DERs is dispatchable at the distribution level and thus the total DER active power curtailment can be minimized by maximizing the total active power injected from all the distribution systems. In this case, the renewable energy consumption indicator can be formulated as follows:

$$
\operatorname{cost}_{D}\left(P_{r e f}^{\text {dist }}\right)=\omega \sum_{k \in D} P_{r e f}^{\text {dist }, k}
$$

where $P_{r e f}^{d i s t, k}$ is the reference of the active injection from distribution system $k$ to the transmission system, $\boldsymbol{D}$ is the set of all distribution systems, $\boldsymbol{P}_{r e f}^{d i s t}$ is a column vector of $P_{r e f}^{d i s t, k}$ for all the $k \in \boldsymbol{D}$, and $\omega$ is a negative constant penalty coefficient, which assigns a higher priority to DERs in the tradeoff considerations with conventional generators according to the equal incremental principle while using $\operatorname{cost}_{D}$ as a penalty term in the objective function [18].

\subsubsection{Line-Loading Indicators under Single-Generator Contingencies}

As the outage loss of faults at the transmission level is much more serious than that at the distribution level, N-1 security indicators should be considered.

The active power flow for line $u$ under the contingency of conventional generator $G_{g}$ is defined as $P_{B r_{u}}^{-G_{g}}$, while the corresponding security threshold is $P_{\mathrm{Br}_{u}}^{\max }$. Since $P_{\mathrm{Br}_{u}}^{-G_{g}}$ cannot directly be measured 
before the contingency actually occurs, an estimation method is proposed based on power transfer distribution factors (PTDFs):

$$
P_{B r_{u}}^{-G_{g}}=P_{B r_{u}}^{\text {tran }}+\sum_{G_{q} \in G^{\text {tran }}} \operatorname{PTDF}_{G_{q}, G_{g}, u} \gamma(q, g) P_{G_{g}}^{\text {tran }}
$$

where $\gamma(q, g)$ is a constant participation factor of generator $G_{q}$ under the contingency of generator $G_{g}$, $P_{B r_{u}}^{t r a n}$ is the current line loading of transmission line $B r_{u}$, and $\mathrm{PTDF}_{G_{q}, G_{g}, u}$ is the line-loading change in the transmission line $B r_{u}$ with 1 p.u. of active power transferred from bus $G_{g}$ to bus $G_{q}$. Both $P_{B r_{u}}^{\text {tran }}$ and $\operatorname{PTDF}_{G_{q}, G_{g}, u}$ can be calculated by the sensitivity of the line loading to bus injection and regarded as constants [19]:

$$
\begin{gathered}
P_{B r_{u}}^{\text {tran }}=\sum_{G_{i} \in G^{\text {tran }}} \frac{\partial P_{B r_{u}}^{\text {tran }}}{\partial P_{G_{i}}^{\text {tran }}} P_{G_{i}}^{\text {tran }}+\sum_{k \in D} \frac{\partial P_{B r_{u}}^{\text {tran }}}{\partial P_{r e f}^{\text {dist }, k}} P_{r e f}^{\text {dist }, k} \\
\operatorname{PTDF}_{q, g, u}=\frac{\partial P_{B r_{u}}^{\text {tran }}}{\partial P_{G_{q}}^{\text {tran }}}-\frac{\partial P_{B r_{u}}^{\text {tran }}}{\partial P_{G_{g}}^{\text {tran }}}
\end{gathered}
$$

Substituting Equations (5) and (6) into Equation (4), the line loadings under the contingency of a single generator are modeled in a linearized form.

\subsubsection{Line-Loading Indicators under Single-Transmission-Line Contingencies}

The active power flow for line $u$ under the contingency of another transmission line $L_{l}$ is defined as $P_{B r_{u}}^{-B r_{l}}$, while the corresponding security threshold is also $P_{B r_{u}}^{\max }$. Since we cannot measure $P_{B r_{u}}^{-\mathrm{Br}_{l}}$ directly, a line outage distribution factor (LODF) is used to calculate it:

$$
P_{B r_{u}}^{-B r_{l}}=P_{B r_{u}}^{t r a n}+\operatorname{LODF}_{u, l} P_{B r_{l}}^{t r a n}
$$

where $\mathrm{LODF}_{u, l}$ is the change in line loading in line $u$ after the outage of line $l$. Denoting the from-bus and the to-bus loadings of line $l$ as $O(l)$ and $D(l)$, respectively, $\operatorname{LODF}_{u, l}$ can be calculated as:

$$
\operatorname{LODF}_{u, l}=\frac{\operatorname{PTDF}_{O(l), D(l), u}}{1-\operatorname{PTDF}_{O(l), D(l), l}} .
$$

Substituting Equations (5) and (8) into Equation (7), the line loadings under the contingency of a single transmission line are also modeled in a linearized form.

\subsubsection{Active Power Loss}

In addition to the above economic and security indicators, the active power loss should also be calculated to ensure power balance.

In contrast to the N-1 indicators, the relationship between the system loss and the bus injections is highly nonlinear and cannot be linearized. However, because the feasible region of real-time dispatch is relatively small under the ramp limitation, the local linearization approximation can be used to describe it:

$$
\Delta P_{\text {loss }}^{\text {tran }}=\sum_{G_{i} \in G^{\text {tran }}} \frac{\partial P_{\text {loss }}^{\text {tran }}}{\partial P_{G_{i}}^{\text {tran }}} \Delta P_{G_{i}}^{\text {tran }}+\sum_{k \in D} \frac{\partial P_{\text {loss }}^{\text {tran }}}{\partial P_{r e f}^{\text {dist }, k}} \Delta P_{r e f}^{\text {dist, },} .
$$

Since the power loss at the current operating point can be calculated directly by summing all the bus injections at the transmission level, the power loss after dispatch can be calculated by Equation (9). 


\subsection{Model Parameter Identification}

Conventionally, the sensitivity factors $\frac{\partial P_{B r u}^{\text {tran }}}{\partial P_{G q}^{\text {tran }}}$ and $\frac{\partial P_{B r u}^{\text {tran }}}{\partial P_{G g}^{\text {tran }}}$ in Equation (6) as well as $\frac{\partial P_{\text {loss }}^{\text {tran }}}{\partial P_{G_{i}}^{\text {Pron }}}$ and $\frac{\partial P_{\text {loss }}^{\text {tran }}}{\partial P_{\text {ref }}^{\text {dist, }}}$ in Equation (9) are calculated from the impedance parameters of the transmission system, which is vulnerable to incorrect impedance parameters and changes in the system topology and operating point. To improve the robustness against these errors, we identify the sensitivity factors from PMU data with a weighted least-squares estimation [4]:

$$
\begin{aligned}
\frac{\partial P_{B r_{u}}^{\text {tran }}}{\partial P^{\text {tran }}} & =\left(\delta P^{\text {tran } T} W \delta P^{\text {tran }}\right)^{-1} \delta P^{\text {tran } T} W \delta P_{B r_{u}}^{\text {tran }} \\
\frac{\partial P_{\text {loss }}^{\text {tran }}}{\partial P^{\text {tran }}} & =\left(\delta P^{\text {tran } T} W \delta P^{\text {tran }}\right)^{-1} \delta P^{\text {tran } T} W \delta P_{\text {loss }}^{\text {tran }}
\end{aligned}
$$

where $P^{\text {tran }}=\left[\left(\boldsymbol{P}_{\text {ref }}^{\text {dist }}\right)^{\mathrm{T}},\left(\boldsymbol{P}_{G}^{\text {tran }}\right)^{\mathrm{T}}\right]^{\mathrm{T}}$ is a column vector of the active power injections of all the transmission buses; $\delta P^{\text {tran }}, \delta P_{B r_{u}}^{\text {tran }}$, and $\delta P_{\text {loss }}^{\text {tran }}$ are time series of the small variations of $P^{\text {tran }}, P_{B r_{u}}^{\text {tran }}$, and $P_{\text {loss }}^{\text {tran }}$ in every PMU sampling period, respectively; and $\boldsymbol{W}$ is a diagonal matrix, which assigns higher weights to closer samples. As this paper does not focus on the technical details of model identification, we refer interested readers to [4] for more details.

\subsection{Formulation and Solution of the Transmission Dispatch Problem}

Since the uncertainty fluctuation in a single interval is almost unpredictable due to the small time scale, real-time dispatch does not provide an optimal motion trail in advance for the operating point, which is in contrast to the conventional dispatch. Instead, we optimize the dispatching decision and move the system to a better operating point at each step by solving Equation (12):

$$
\min _{P_{\text {ref }}^{\text {dist }}, P_{G}^{\text {tran }}} \operatorname{cost} G\left(P_{G}^{\text {tran }}\right)+\operatorname{cost}_{D}\left(P_{\text {ref }}^{\text {dist }}\right)
$$

s.t.

$$
\begin{aligned}
& \sum_{G_{i} \in G^{\text {tran }}} P_{G_{i}}^{\text {tran }}+\sum_{k \in D} P_{r e f}^{\text {dist }, k}-P_{\text {loss }}^{\text {tran }}=0 \\
& \begin{array}{l}
\boldsymbol{P}_{G_{i}, \text { min }}^{\text {tran }}<\boldsymbol{P}_{G_{i}}^{\text {tran }}<\boldsymbol{P}_{G_{i}, \text { max }}^{\text {tran }}, \quad \forall G_{i} \in G^{\text {tran }} \\
\left|\Delta \boldsymbol{P}_{G_{i}}^{\text {tran }}\right|<\boldsymbol{P}_{G_{i}, \text { ramp }}^{\text {tran }}
\end{array} \\
& P_{\text {min }}^{\text {dist }, k}<P_{\text {ref }}^{\text {dist }, k}<P_{\text {max }}^{\text {dist }, k}, \forall k \in D \\
& \left|P_{B r_{u}}^{-B r_{l}}\right|<P_{B r_{u}}^{\text {max }}, \forall B r_{l} \in B r \\
& \left|P_{B r_{u}}^{-G_{g}}\right|<P_{B r_{u}}^{m a x}, \forall G_{g} \in G^{\text {tran }}, \quad \forall B r_{u} \in B r
\end{aligned}
$$

where $P_{\max }^{d i s t, k}$ and $P_{\min }^{\text {dist, } k}$ are calculated at the distribution level, as described in Section 3.

Substituting Equations (4), (5) and (9) into Equation (12), the transmission level problem is transformed into a quadratic form that can be quickly optimized in real time by any quadratic built-in solver in the centralized controller.

\section{Real-Time Dispatch Strategy at the Distribution Level}

At the distribution level, the active power injection of all the DERs should be regulated to maintain the total active power injected into the transmission system at the reference, which is optimized at the transmission level. To mitigate the impact of DER active power injections on the bus voltages and utilize the potential of the distribution system to inject active power into the transmission system, the reactive power of the DERs should be optimized to meet the bus voltage limitations and achieve loss 
minimization. Moreover, the regulating range of $P_{r e f}^{d i s t, k}$ also needs to be calculated and provided to the transmission system to assist in transmission dispatch.

Due to the relatively poor measurement and communication infrastructure and the higher privacy requirements, the dispatch at the distribution level should avoid dependency on PMU data, real-time consumer demand, and centralized control as is done at the transmission level.

Taken together, a decentralized coordinated control strategy, which can coordinate the active and reactive power generation of all the DERs with limited measurements and communication and upload necessary information to the transmission system, is proposed in Section 3.

For the DERs in the $k$ th distribution system, the following control laws are implemented.

\subsection{Active Power Control}

For each bus $i$, if there is no DER bus along path $p_{i, j}$ except for $i$ and $j$ themselves, we can state that bus $j$ is a neighbor of bus $i$, or neighbors $i$. Denoting the set of all buses neighboring $i$ as the neighborhood $N(i)$, we have $j \in N(i)$ when $j$ neighbors $i$.

Thus, for each DER $G_{i} \in G^{\text {dist, },}$, an average-consensus-based active power control law is implemented to achieve power sharing while maintaining the common coupling point (PCC) power injection into the transmission level:

$$
\begin{gathered}
\vartheta_{P C C}^{\text {dist }, k}=P_{0}^{\text {dist }, k}+P_{r e f}^{\text {dist }, k} \\
\vartheta_{G_{i}, \text { share }}^{\text {dist },}=\sum_{G_{j} \in N\left(G_{i}\right)} \frac{P_{G_{j}}^{d i s t, k}}{P_{G_{j}, m a x}^{d i s t, k}}-\frac{P_{G_{i}}^{d i s t, k}}{P_{G_{i}, \text { dist }, k a x}} \\
\Delta P_{G_{i}}^{\text {dist }, k}=K_{1}^{G_{i}} \vartheta_{P C C}^{\text {dist }, k}+K_{2}^{G_{i}} \vartheta_{G_{i}, \text { share }}^{\text {dist }, k}
\end{gathered}
$$

where $P_{G_{i}}^{d i s t, k}$ is the active power generation of DER $G_{i} ; P_{G_{j}, \max }^{d i s t, k}$ is the current maximum active generation of DER $G_{i} ; \vartheta_{P C C}^{\text {dist,k }}$ is the PCC active power deviation broadcasted from the PCC; $P_{0}^{\text {dist }, k}$ is the PCC active power of the $k$ th distribution system; $P_{r e f}^{d i s t, k}$ is the reference value of the active injection from the $k$ th distribution system to the transmission system; $\vartheta_{G_{i}, \text { share }}^{\text {dist }, k}$ is a correction for power sharing; $\frac{P_{G_{j}}^{\text {dist }, k}}{P_{G_{j}, m a x}^{\text {dist }, k}}$ is the active power sharing proportion of DER $G_{j}$, which is gathered by the intercommunications among the neighboring DERs; and $K_{1}^{G_{i}}$ and $K_{2}^{G_{i}}$ are constant controller gains, which can be set according to the condition of convergence discussed in [14]. When the consensus of Equation (15) is reached, the active power injected from the $k$ th distribution system to the transmission system will be equal to $P_{r e f}^{d i s t, k}$, while the active power will be shared proportionally based on DER capacity.

\subsection{Reactive Power Control}

Since the branches in a distribution system often have similar $\mathrm{R} / \mathrm{X}$ ratios, the average impedance angle $\theta^{k}$ of all distribution lines is used to approximate the specific impedance angle of each distribution line in the $k$ th distribution system. Denoting $r_{0}^{\text {dist }, k}=\cos \theta^{d i s t, k}, x_{0}^{d i s t, k}=\sin \theta^{\text {dist }, k}$, and $z_{0}^{\text {dist }, k}=$ $r_{0}^{d i s t, k}+\mathrm{i} x_{0}^{d i s t, k}$, the total branch impedance along each path $p_{i, j}$ in distribution system $k$ can then be approximated as $z_{p_{i, j}}^{\text {dist }, k}=\left|z_{p_{i, j}}^{\text {dist }, k}\right| z_{0}^{\text {dist }, k}$, where $\left|z_{p_{i, j}}^{\text {dist }, k}\right|$ is the magnitude of $z_{p_{i, j}}^{\text {dist }, k}$. Defining the path from the PCC to buses $i$ and $j$ as $p_{0, i}$ and $p_{0, j}$, respectively, the total impedance magnitude of the common part can then be denoted by $\left|z_{p_{0, i} \cap p_{0, j}}^{\text {dist }}\right|$. Moreover, $y_{i, j}^{\text {dist }, k}$ is defined as the admittance between buses $i$ and $j$. In this case, for each DER $G_{i} \in G^{d i s t, k}$, the reactive power reference can be dispatched as follows:

$$
Q_{G_{i}, \Delta P}^{d i s t, k}=-\tan \theta^{d i s t, k} \Delta P_{G_{i}}^{d i s t, k}
$$




$$
\begin{aligned}
& Q_{G_{i}, l o s s}^{\text {dist }, k}=-\sum_{G_{h} \in N\left(G_{i}\right)} y_{i h}^{\text {dist }, k}\left(-\left|z_{p_{0, O_{i}}}^{\text {dist }, k}\right| \sum_{G_{l} \in N\left(G_{i}\right)} Q_{G_{l} \rightarrow G_{i}}^{\text {dist }, k}+\left(V_{O_{i}}^{\text {dist }, k}-V_{G_{h}}^{\text {dist }, k}\right.\right. \\
& \left.-\sum_{G_{l} \in N\left(G_{i}\right)}\left(x_{0} Q_{G_{l} \rightarrow G_{i}}+r_{0} P_{G_{l} \rightarrow G_{i}}\right)\left|z_{p_{O_{i}, G_{l}} \cap i s t, k}^{d i p_{i}, G_{h}}\right|\right) /\left(x_{0}\right. \\
& \left.\left.+r_{0} \frac{\sum_{G_{G} \in N\left(G_{i}\right)} P_{G_{l} \rightarrow G_{i}}}{\sum_{G_{l} \in N\left(G_{i}\right)} Q_{G_{l} \rightarrow G_{i}}}\right)\right) \\
& Q_{G_{i}, \text { cons }}^{\text {dist } k}=\sum_{G_{h} \in N\left(G_{i}\right)} y_{G_{i}, G_{h}}^{\text {dist }, k}\left(\mu_{G_{h}, \max }^{\text {dist }, k}-\mu_{G_{h}, \text { min }}^{\text {dist } k}\right. \\
& \left.+x_{0}^{d i s t, k} \sum_{L_{j} \in N\left(G_{i}\right)}\left|z_{p_{0, G_{h}} \cap p_{0, L_{j}}}^{\text {dist },}\right|\left(\lambda_{L_{j}, \max }^{\text {dist } k}-\lambda_{L_{j}, \min }^{\text {dist } k}\right)\right)+x_{0}^{\text {dist, }, k}>\left(\lambda_{G_{i}, \max }^{\text {dist } k}\right. \\
& \left.-\lambda_{G_{i}, \text { min }}^{\text {dist,k}}\right) \\
& \widetilde{Q}_{G_{i}}^{\text {dist }, k}=Q_{G_{i}, \Delta P}^{d i s t, k}+Q_{G_{i}, \text { ons }}^{\text {dist },}+Q_{G_{i}, l o s s}^{\text {dist } k}
\end{aligned}
$$

where $\widetilde{Q}_{G_{i}}^{\text {dist }, k}$ is the reactive output of DER $G_{i}$ dispatched in the current interval; $Q_{G_{i}, \Delta P}^{\text {dist }, k}$ is the reactive power to offset the voltage deviation caused by the active dispatch in the same interval; $Q_{G_{i}, l o s s}^{\text {dist } k}$ is the reactive power to minimize the total system active loss, where $O_{i}$ is the root node of neighborhood $N\left(G_{i}\right)$, and $P_{G_{l} \rightarrow G_{i}}$ and $Q_{G_{l} \rightarrow G_{i}}$ are the total active and reactive branch flows, respectively, transmitted from DER $G_{l}$ into neighborhood $N\left(G_{i}\right)$, which can be measured at $G_{l}$ and gathered by $G_{i}$ with the neighboring intercommunications; and $Q_{G_{i}, \text { cons }}^{\text {dist } k}$ is a term used to handle the constraints, where $\mu$ and $\lambda$ are positive intermediate variables stored in each DER, the update rules of which for each DER $G_{i} \in G^{d i s t, k}$ are given as:

$$
\begin{aligned}
& \Delta \mu_{G_{i,}, \max }^{\text {dist }}=\gamma\left(Q_{G_{i}}^{\text {dist }, k}-Q_{G_{i}, \text { max }}^{\text {dist }, k}\right) \\
& \Delta \mu_{G_{i}, \min }^{\text {dist }}=\gamma\left(Q_{G_{i}, \min }^{\text {dist }, k}-Q_{G_{i}}^{\text {dist }, k}\right) \\
& \Delta \lambda_{G_{i}, \max }^{\text {dist }, k}=\gamma\left(V_{G_{i}}^{\text {dist }, k}-V_{G_{i}, \max }^{\text {dist }, k}\right) \\
& \Delta \lambda_{G_{i}, \min }^{\text {dist } k}=\gamma\left(V_{G_{i}, \min }^{\text {dist } k}-V_{G_{i}}^{d i s t, k}\right) \\
& \Delta \lambda_{L_{j}, \max }^{\text {dist }, k}=\gamma\left(V_{L_{j}}^{\text {dist }, k}-V_{L_{j}, \max }^{\text {dist } k}\right) \\
& \Delta \lambda_{L_{j}, \min }^{\text {dist }, k}=\gamma\left(V_{L_{j}, \min }^{\text {dist }}-V_{L_{j}}^{\text {dist }, k}\right), \quad \forall L_{j} \in N\left(G_{i}\right)
\end{aligned}
$$

where $V_{L_{j}}^{\text {dist }, k}$ is the voltage magnitude of load $L_{j}$, which can be broadcasted from the neighboring bus loads; $V_{L_{j}}, V_{L_{j}, \text { min }}^{\text {dist }}$, and $V_{L_{i}, \text { max }}^{\text {dist } k}$ are the voltage and its limitations of load $L_{j} ; V_{G_{i}, \text { min }}^{\text {dist } k}$ and $V_{G_{i}, \text { max }}^{\text {dist, }}$ are the voltage limitations of DER $G_{i} ; Q_{G_{i}, \text { min }}^{\text {dist }}$ and $Q_{G_{i}, \text { max }}^{\text {dist, }}$ are the reactive power limitations of the inverters, which can be calculated by the installed capacity of the inverters and their active power injections; and $\gamma$ is a constant controller gain. All the intermediate variables need to be corrected to 0 if they become negative after being updated according to Equation (20).

With the alternate execution of Equations (19) and (20), $Q_{G}^{\text {dist, } k}$ converges to Equation (21) according to a series of theoretical derivations (under generalization of the LinDistFlow model $[20,21]$ to radial systems) and numerical simulations [22-24].

$$
\min _{Q_{G}^{d i s t, k}} P_{\text {list }, k}^{\text {loss }}
$$

s.t.

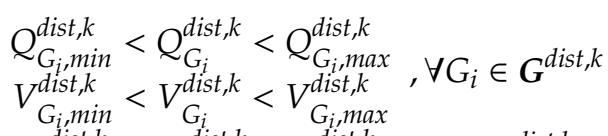

$$
\begin{aligned}
& V_{L_{i}, \text { min }}^{d i s t, k}<V_{L_{i}}^{\text {dist }, k}<V_{L_{i}, \text { max }^{\prime}}^{\text {dist }}, \forall L_{i} \in L^{\text {dist }, k}
\end{aligned}
$$


where $P_{\text {list }}^{\text {dis }}$ is the total active line loss in the $k$ th distribution system, and $L^{d i s t, k}$ is the set of all load buses in the th distribution system. The decision vector $Q_{G}^{\text {dist, } k}$ is a vector of the reactive power injections of all the DERs in the $k$ th distribution system. Minimizing the total active line loss can expand the dispatchable range of the PCC active power. In this case, reducing the active loss $P_{\text {loss }}^{d i s t, k}$ can increase the ceiling of $P_{0}^{d i s t, k}$, which will provide a wider dispatchable range for the transmission system.

It is worth mentioning that the term $Q_{G_{i}, \Delta P}^{\text {dist, }}$ does not contribute to solving Equation (21), which will be 0 if there is no need to dispatch the local active power.

\subsection{The Dispatchable Region of the PCC Active Power}

To calculate the total active dispatch potential of all the DERs in the distribution system, the PCC will broadcast a command signal to all the DERs to start the estimation process. In this process, each $D E R G_{i} \in G^{\text {dist }, k}$ will separately estimate the average generation potential of the DERs in the distribution system as $P_{G_{i}, e s t i}^{\text {dist } k}=P_{G_{i}, \max }^{\text {dist } k}$ and then correct the estimation with the average-consensus-based method:

$$
\Delta P_{G_{i}, e s t i}^{d i s t, k}=K_{3}^{G_{i}} \sum_{G_{j} \in N\left(G_{i}\right)} P_{G_{j}, e s t i}^{d i s t, k}-P_{G_{i}, e s t i}^{d i s t, k}
$$

where $K_{3}^{G_{i}}$ is a constant controller gain and all $\Delta P_{G_{i}, e s t i}^{d i s t, k}$ will converge to the actual value of the average generation potential:

$$
P_{a v e, G_{\max }}^{d i s t, k}=\frac{\sum_{G_{h} \in G^{d i s t, k}} P_{G_{h}, \text { max }}^{\text {dist } k}}{\operatorname{num}\left(G^{\text {dist }, k}\right)}
$$

where $n u m\left(G^{\text {dist }, k}\right)$ is the number of DERs in the distribution system, which is registered in advance to the PCC.

Since the PCC is connected to the intercommunication system of the distribution system, it can receive the values of $P_{a v e, G_{\max }}^{d i s t, k}$ and $\Lambda^{d i s t, k}$ from the neighboring DERs. In this case, $P_{\min }^{\text {dist }, k}$ and $P_{\max }^{\text {dist }, k}$ can be calculated as:

$$
\begin{gathered}
P_{\text {min }}^{\text {dist }, k}=P_{\text {ref }}^{\text {dist }, k}-\operatorname{num}\left(G^{\text {dist }, k}\right) P_{\text {ave }, G_{\max }}^{\text {dist },} \Lambda^{\text {dist }, k} \\
P_{\text {max }}^{\text {dist }, k}=P_{\text {ref }}^{\text {dist }, k}+\operatorname{num}\left(G^{\text {dist }, k}\right) P_{\text {ave }, G_{\text {max }}}^{\text {dist }, k}\left(1-\Lambda^{\text {dist }, k}\right)
\end{gathered}
$$

where $\Lambda^{\text {dist }, k}=\frac{\sum_{G_{h} \in G^{d i s t, ~}} P_{G_{h}}^{d i s t, k}}{\sum_{G_{h} \in G^{d i s t, k}, k} P_{G_{h}, k}^{\text {max }}}$, which can be easily obtained from any DER after the consensus of Equation (15) is reached.

\section{Case Study}

The IEEE 39-bus transmission system (the system model of which can be found in [25]) coupled with modified IEEE-33 bus distribution systems (the system model of which can be found in [24]) is adopted to evaluate the effectiveness of the proposed real-time dispatch strategy for coupled transmission and distribution systems. Ten 33-bus distribution systems are connected to bus 11 of the 39-bus transmission system, while each 33-bus system is connected with eight dispatchable DERs at distribution buses $9,11,13,21,22,25,27$, and 29. The total active load demand of all 33-bus systems is 60.3 MW, while the prediction value of the total DER active generation of all the 33-bus systems used in the conventional dispatch with larger time scales is $40.3 \mathrm{MW}$, and the actual value leapt to $70.3 \mathrm{MW}$. The initial load demand of each bus at the transmission level is shown in Figure 1, while the initial active injections of the conventional generators are shown in Figure 2. 


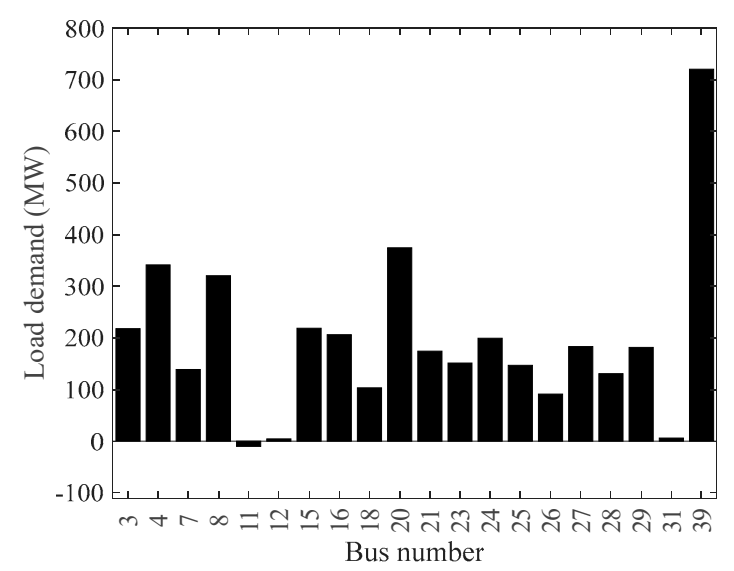

Figure 1. The initial load demands in the transmission system.

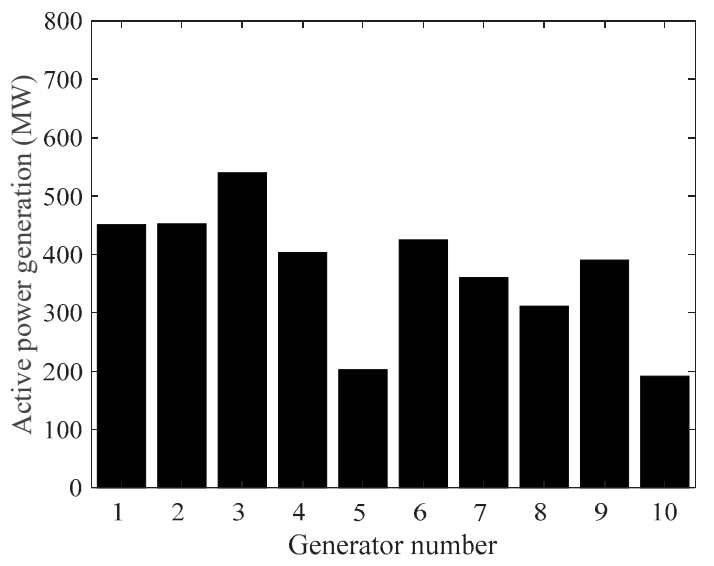

Figure 2. The initial active generation of the conventional generators.

In this case, the operating point will deviate from the security region if all the DERs work in the MPPT mode. The branch flow between buses 6 and 11 will be $105.4 \%$ of its limitation if the transmission line between buses 13 and 14 is under contingency. The user behavior and meteorological conditions remain constant during the simulation, which lasts for $10 \mathrm{~min}$, and thus, the out-of-limit value of the $\mathrm{N}-1$ indicator can be recovered only by dispatching conventional generators in the transmission system and the DERs in the distribution system. Moreover, because the distribution systems are all under heavy loads, reactive dispatch is required to maintain bus voltage.

To evaluate the necessity of the proposed coordination strategy for distribution and transmission dispatch, benchmarks 1 and 2 are used. In benchmark 1 , all the DERs work in the maximum power point tracking (MPPT) mode so that the renewable energy will be completely consumed. In benchmark 2 , the DERs reduce their active output to the predicted value. In both benchmarks, the conventional generators at the transmission level are dispatched by the measurement-based real-time dispatch strategy proposed in [4], which is similar to the transmission dispatch method in this paper but does not consider the DERs. The control strategy developed in [14] is adopted in both benchmarks to achieve active power sharing and voltage control. The PCC reactive references in the benchmarks are all set to 0 according to the principle of locally reactive balances. The dispatch interval at the transmission level is $1 \mathrm{~min}$, while the distribution dispatch interval is set to $6 \mathrm{~s}$. The simulation results, which are shown in Figures 3-7, reveal the following advantages of the proposed method: 


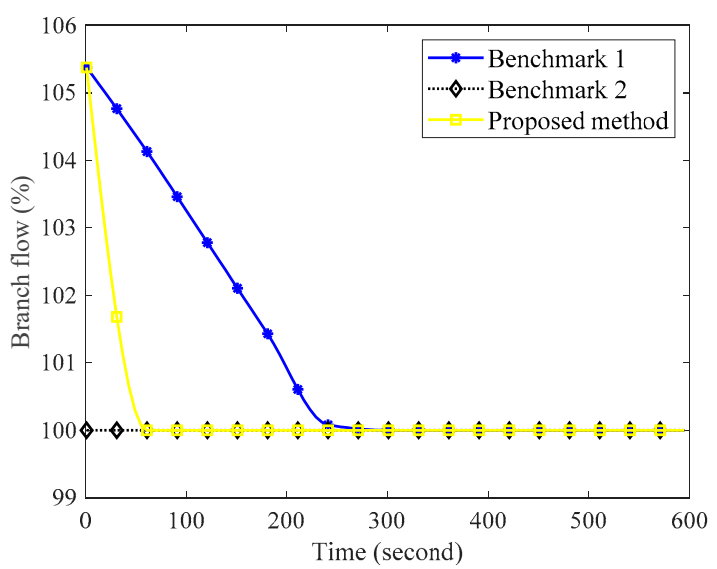

Figure 3. The branch flow between buses 6 and 11 under the contingency of the branch between buses 13 and 14.

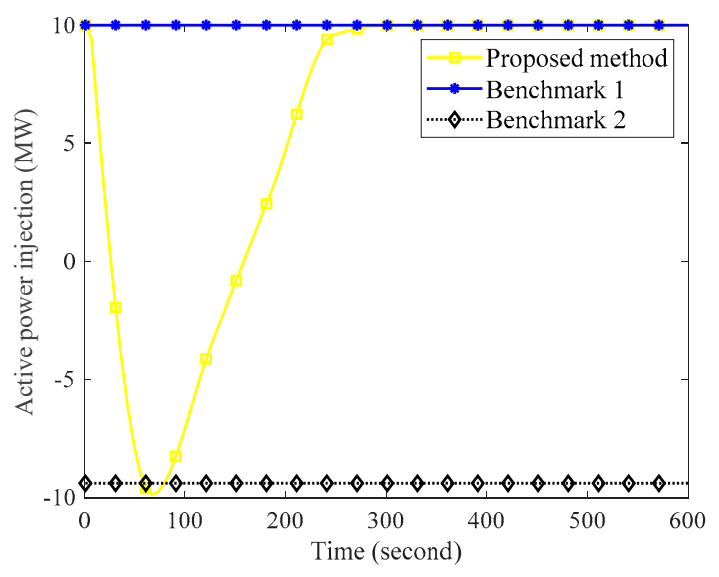

Figure 4. The total active injection of all 33-bus systems.

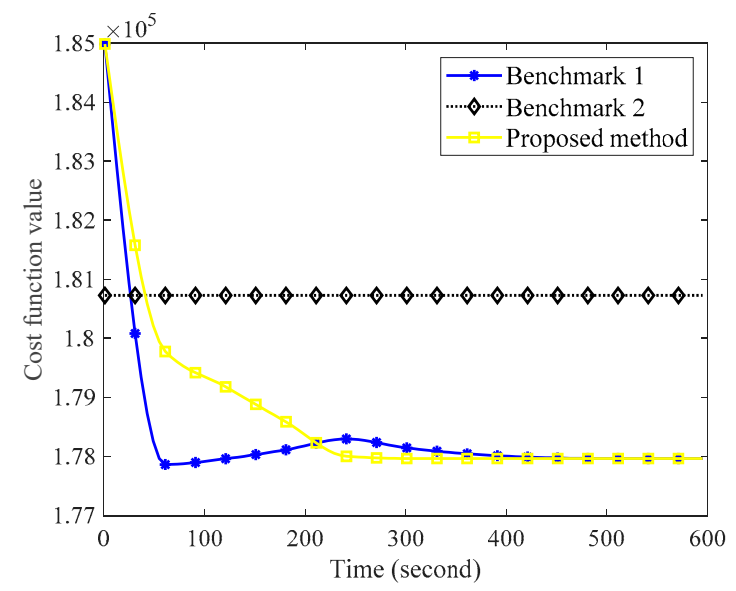

Figure 5. The cost function value. 


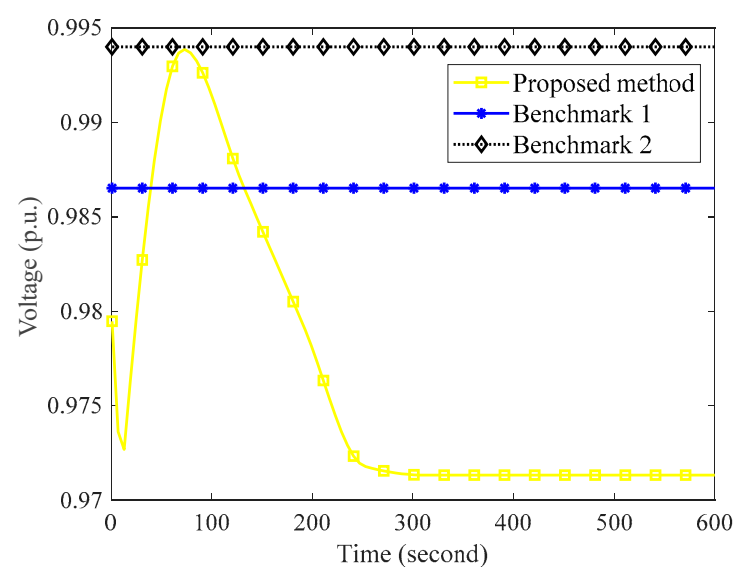

Figure 6. The lowest distribution bus voltage in the 33-bus systems.

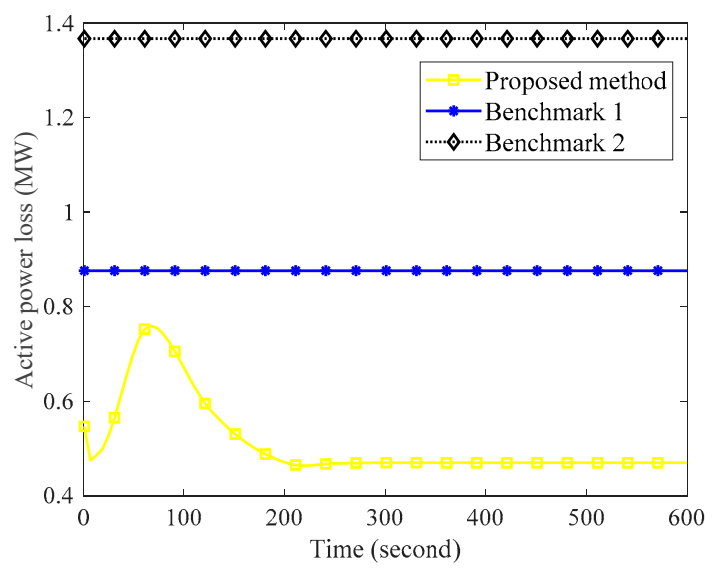

Figure 7. The total active loss of all 33-bus systems.

\subsection{The Proposed Method Can Quickly Eliminate the Security Issue Using DERs}

As shown in Figure 3, more than 5 min are required in benchmark 1 to restore the out-of-limit N-1 indicator because it dispatches only the conventional generators to handle the security problem. It will be very dangerous if something happens on the branch between bus 13 and bus 14 within this period. To reduce this potential risk, the proposed method decreases the active power injected from the 33-bus systems into the transmission level, as shown in Figure 4, which can solve the security problem within $1 \mathrm{~min}$

\subsection{The Proposed Method Can Quickly Recover Renewable Energy Consumption Right after the Security Problem Is Solved}

The method in benchmark 2 can also avoid the security problem as it never allows the distribution system to inject more active power into the transmission system than the dispatched value based on prediction. However, this method is too conservative and may lead to persistent renewable energy curtailment, as shown in Figure 4. The proposed method reduces the DER generation only at the beginning and the DERs can return to the MPPT mode after the conventional generators reach a new suitable operating point that can completely consume the DER injection from all 33-bus systems without leading to a security problem. As shown in Figure 5, the proposed method can finally maintain a cost similar to that of benchmark 1, which never reduced DER generation. 
4.3. The Proposed Method Can Maintain a Stable Distribution Bus Voltage during the Active Power Dispatch and Then Reduce the Active Power Loss

The lowest distribution bus voltage in the 33-bus systems is shown in Figure 6. Since the benchmarks do not change their DER generation, the voltage will of course be smooth and steady. Although the proposed method needs to quickly reduce the active generation of DERs, the reactive control can avoid an excessive voltage drop. Moreover, the active loss of the proposed method is much lower than that in benchmark 1, as shown in Figure 7, even though the active generation of the DERs is the same in the last $5 \mathrm{~min}$.

\subsection{The Proposed Method Can Provide Sufficient Response Speed to Maintain a Good Real-Time Performance}

Two $1.80 \mathrm{GHz}$ CPUs are used to run the power system simulation and the proposed algorithms. The time cost of all of the calculations for the transmission system is less than $0.5 \mathrm{~s}$ for any calculation period, which is fast enough to allow the method to work in real-time. For the distribution systems, the calculation time for each DER in each calculation period is much smaller than $0.1 \mathrm{~s}$, which is also fast enough.

For larger systems, since the computational tasks in distribution system are assigned to all the DERs, the calculation time for the controller of each DER will not increase with the system scale. For the transmission system, since Equation (12) is a quadratic programming problem, developed algorithms for quadratic programming problems can be used to ensure real-time performance.

\section{Conclusions}

A decentralized coordinated real-time dispatch strategy for coupled transmission and distribution systems is proposed in this paper. A measurement-based centralized structure was used at the transmission level to take full advantage of the advanced IT infrastructure to obtain more robustness against model uncertainties, while a distributed structure with limited broadcast communication was used at the distribution level to adapt to the lack of communication. The case study revealed that the proposed dispatch strategy quickly resolved the security problem at the transmission level depending on the high ramp rate of the DERs in the distribution systems and consumed more of the active power injected by the DERs by dispatching conventional generators in the transmission systems. The voltage fluctuation caused by the DER active generation was relieved by controlling the DER reactive power, which could also reduce the active power loss at the distribution level. The proposed calculation methods were fast enough to maintain real-time performance in the study cases, while the calculation time did not rise sharply in a larger system for the distributed characteristics and simple structures of the proposed methods. In this case, the proposed distributed and cooperative framework could be used to achieve the real-time dispatch of coupled transmission and distribution systems with DERs. The future direction of this research is to consider the small-signal-stability constraints in the transmission system in the dispatch framework and provide a new method to reduce constraint violations during the dispatch process.

Author Contributions: The authors confirm that the contributions to this paper are as follows: Study conception and design: L.G.; theoretical derivation: L.Z.; simulation verification: L.Z.; draft manuscript preparation: L.Z. All authors have read and agreed to the published version of the manuscript.

Funding: This work was supported by the National Natural Science Foundation of China under Grants 51761145106.

Acknowledgments: The financial support of the National Natural Science Foundation of China is gratefully acknowledged.

Conflicts of Interest: The authors declare that there are no conflict of interest. 


\section{References}

1. Karimi, M.; Mokhlis, H.; Naidu, K.; Uddin, S.; Bakar, A.H.A. Photovoltaic penetration issues and impacts in distribution network-A review. Renew. Sustain. Energy Rev. 2016, 53, 594-605. [CrossRef]

2. Haque, M.M.; Wolfs, P. A review of high PV penetrations in LV distribution networks: Present status, impacts and mitigation measures. Renew. Sustain. Energy Rev. 2016, 62, 1195-1208. [CrossRef]

3. Perez-Arriaga, I.J. The transmission of the future: The impact of distributed energy resources on the network. IEEE Power Energy Mag. 2016, 14, 41-53. [CrossRef]

4. Van Horn, K.E.; Domínguez-García, A.D.; Sauer, P.W. Measurement-based real-time security-constrained economic dispatch. IEEE Trans. Power Syst. 2016, 31, 3548-3560. [CrossRef]

5. Zhang, W.; Liu, W.; Wang, X.; Liu, L.; Ferrese, F. Online optimal generation control based on constrained distributed gradient algorithm. IEEE Trans. Power Syst. 2015, 30, 35-45. [CrossRef]

6. Zegers, A.; Brunner, H. TSO-DSO Interaction: An Overview of Current Interaction between Transmission and Distribution System Operators and an Assessment of Their Cooperation in Smart Grids, September 2014. Available online: http://smartgrids.no/wp-content/uploads/sites/4/2016/01/ISGAN-TSO-DSO-interaction.pdf (accessed on 18 September 2020).

7. Gerard, H.; Puente, E.I.R.; Six, D. Coordination between transmission and distribution system operators in the electricity sector: A conceptual framework. Utilities Policy 2018, 50, 40-48. [CrossRef]

8. Mirhassani, S.; Ong, H.C.; Chong, W.T.; Leong, K.Y. Advances and challenges in grid tied photovoltaic systems. Renew. Sustain. Energy Rev. 2015, 49, 121-131. [CrossRef]

9. Lin, C.; Wu, W.; Shahidehpour, M. Decentralized AC optimal power flow for integrated transmission and distribution grids. IEEE Trans. Smart Grid 2019, 11, 2531-2540. [CrossRef]

10. Li, Z.; Sun, H.; Guo, Q.; Wang, J.; Liu, G. Generalized master-slave-splitting method and application to transmission-distribution coordinated energy management. IEEE Trans. Power Syst. 2019, 34, 5169-5183. [CrossRef]

11. Mulligan, M.A.L.K.; Wicker, S.B. Inferring Personal Information from Demand-Response Systems. IEEE Secur. Priv. 2010, 8, 11-20.

12. Sossan, F.; Namor, E.; Cherkaoui, R.; Paolone, M. Achieving the Dispatchability of Distribution Feeders Through Prosumers Data Driven Forecasting and Model Predictive Control of Electrochemical Storage. IEEE Trans. Sustain. Energy 2016, 7, 1762-1777. [CrossRef]

13. Valverde, G.; Shchetinin, D.; Hug-Glanzmann, G. Coordination of Distributed Reactive Power Sources for Voltage Support of Transmission Networks. IEEE Trans. Sustain. Energy 2019, 10, 1544-1553. [CrossRef]

14. Yang, Z.; Li, Y.; Xiang, J. Coordination control strategy for power management of active distribution networks. IEEE Trans. Smart Grid 2019, 10, 5524-5535. [CrossRef]

15. Arnold, D.B.; Sankur, M.D.; Negrete-Pincetic, M.; Callaway, D.S. Model-Free Optimal Coordination of Distributed Energy Resources for Provisioning Transmission-Level Services. IEEE Trans. Power Syst. 2018, 33, 817-828. [CrossRef]

16. Li, Z.; Guo, Q.; Sun, H.; Wang, J. Coordinated Transmission and Distribution AC Optimal Power Flow. IEEE Trans. Smart Grid 2018, 9, 1228-1240. [CrossRef]

17. Tang, K.; Dong, S.; Zhu, C.; Song, Y. Affine Arithmetic-Based Coordinated Interval Power Flow of Integrated Transmission and Distribution Networks. IEEE Trans. Smart Grid 2020, 11, 4116-4132. [CrossRef]

18. Xu, T.; Wu, W.; Zheng, W.; Sun, H.; Wang, L. Fully Distributed Quasi-Newton Multi-Area Dynamic Economic Dispatch Method for Active Distribution Networks. IEEE Trans. Power Syst. 2018, 33, 4253-4263. [CrossRef]

19. Wood, B.; Wollenberg, B.; Sheblé, G. Power Generation, Operation, and Control, 3rd ed.; John Wiley and Sons, Inc.: Hoboken, NJ, USA, 2014.

20. Sulc, P.; Backhaus, S.; Chertkov, M. Optimal distributed control of reactive power via the alternating direction method of multipliers. IEEE Trans. Energy Convers. 2014, 29, 968-977. [CrossRef]

21. Low, S.H. Convex relaxation of optimal power flow-Part I: Formulations and equivalence. IEEE Trans. Control Netw. Syst. 2014, 1, 15-27. [CrossRef]

22. Bolognani, S.; Carli, R.; Cavraro, G.; Zampieri, S. Distributed reactive power feedback control for voltage regulation and loss minimization. IEEE Trans. Autom. Control 2015, 60, 966-981. [CrossRef]

23. Cavraro, G.; Carli, R. Local and Distributed Voltage Control Algorithms in Distribution Networks. IEEE Trans. Power Syst. 2018, 33, 1420-1430. [CrossRef] 
24. Zhong, L.; Guan, L.; Zhang, J.; Gong, Y.; Chung, C.Y. Distributed reactive power regulation considering load voltage and power loss. IEEE Access 2020, 8, 24334-24343. [CrossRef]

25. Athay, T.; Podmore, R.; Virmani, S. A practical method for the direct analysis of transient stability. IEEE Trans. Power Appl. Syst. 1979, PAS-98, 573-584. [CrossRef]

(C) 2020 by the authors. Licensee MDPI, Basel, Switzerland. This article is an open access article distributed under the terms and conditions of the Creative Commons Attribution (CC BY) license (http://creativecommons.org/licenses/by/4.0/). 